This is an electronic reprint of the original article. This reprint may differ from the original in pagination and typographic detail.

Author(s): Banichuk, Nikolay; Barsuk, Alexandr; Ivanova, Svetlana; Jeronen, Juha; Makeev, Evgeni; Tuovinen, Tero

Title: $\quad$ Vibrations of a continuous web on elastic supports

Year: $\quad 2018$

Version:

Please cite the original version:

Banichuk, N., Barsuk, A., Ivanova, S., Jeronen, J., Makeev, E., \& Tuovinen, T. (2018). Vibrations of a continuous web on elastic supports. Mechanics Based Design of Structures and Machines, 46(1), 1-17. https://doi.org/10.1080/15397734.2016.1261034

All material supplied via JYX is protected by copyright and other intellectual property rights, and duplication or sale of all or part of any of the repository collections is not permitted, except that material may be duplicated by you for your research use or educational purposes in electronic or print form. You must obtain permission for any other use. Electronic or print copies may not be offered, whether for sale or otherwise to anyone who is not an authorised user. 


\title{
Vibrations of a continuous web on elastic supports
}

Nikolay Banichuk ${ }^{1}$, Alexandr Barsuk ${ }^{2}$, Svetlana Ivanova ${ }^{1}$, Juha Jeronen $^{3}$, Evgeni Makeev ${ }^{1}$, and Tero Tuovinen ${ }^{3}$

\author{
${ }^{1}$ Institute for Problems in Mechanics RAS, Moscow, Russian Federation \\ ${ }^{2}$ Department of Theoretical Physics, State University of Moldova, Chisinau, Moldova \\ ${ }^{3}$ Department of Mathematical Information Technology, University of Jyväskylä, Agora, Finland
}

\begin{abstract}
We consider an infinite, homogeneous linearly elastic beam resting on a system of linearly elastic supports, as an idealized model for a paper web in the middle of a cylinder-based dryer section. We obtain closed-form analytical expressions for the eigenfrequencies and the eigenmodes. The frequencies increase as the support rigidity is increased. Each frequency is bounded from above by the solution with absolutely rigid supports, and from below by the solution in the limit of vanishing support rigidity. Thus in a real system, the natural frequencies will be lower than predicted by commonly used models with rigid supports.
\end{abstract}

KEYWORDS: Dynamics of Machines; Elasticity/Viscoelasticity; Structural Dynamics/Vibration

Received 5 February 2016; Revised 09 November 2016; Accepted 11 November 2016

Juha Jeronen juha.jeronen@jyu.fi Department of Mathematical Information Technology, University of Jyväskylä, P.O. Box 35, Agora, FI 40014, Finland. 


\section{INTRODUCTION}

In our previous studies (see, e.g., 3-8), we have considered mathematical modelling of the mechanical aspects of the paper making process, using analytical and numerical approaches. The processing of paper, steel, fabric or rubber, and looping systems such as band saws and timing belts, all belong to the class of systems known as axially moving materials. Typically the material is a continuous thin sheet, travelling across a rectangular span, with two opposite edges supported by rollers, and the other two opposite edges free of tractions.

The most often used models for an axially moving continuous material are travelling flexible strings, membranes, beams, and plates. The string and beam models are applicable also to thin sheets undergoing cylindrical deformation.

The research field of moving materials can be traced back to (34). Among the first English-language papers on moving materials were (31) and (25). All these studies considered axially moving ideal strings. The analytical solution describing the free vibrations of the axially moving ideal string was derived by (36). Dynamics and stability considerations were first reviewed in (27).

The effects of axial motion of the material on its frequency spectrum and eigenfunctions were investigated for strings by (2) and for beams by (33). It was shown that the natural frequency of each mode decreases when the transport speed increases, and that the travelling string and beam both experience divergence instability at a sufficiently high speed. 
Travelling beams have been further analyzed by (28), and (16). In the latter, an approximate analytical expression was derived for the eigenfrequencies of a moving beam with small flexural stiffness. Arbitrary excitations and initial conditions were analyzed with the help of modal analysis and a Green's function method in (44). Travelling strings and beams on an elastic foundation have been investigated by, e.g., (9), (30), (43), and (29). Travelling viscoelastic beams with timedependent speed were recently considered by (11).

The loss of stability was studied with an application of dynamic and static approaches in (42). It was shown via numerical analysis that in the all cases instability occurs when the frequency is zero, and the critical velocity coincides with the corresponding velocity obtained from a static analysis. Similar results were obtained for travelling plates by (21).

For exploring further, extensive literature reviews about the dynamics of axially moving continua can be found in (24) and in (5). For axially moving strings specifically, see the excellent review article by (10).

For classical stationary (as opposed to axially moving) beams, a simple unified approach for the vibration analysis of a generally supported beam is presented in $(17,19)$. A flexural displacement of the beam is sought as the linear combination of a Fourier series and an auxiliary polynomial function. (18) provides an alternative discretization scheme based on the Galerkin method. (20) concentrates on vibration analysis using general boundary conditions and the Rayleigh-Ritz 
method, to determine the modal characteristics of a rectangular plate with general elastic supports along its edges.

(13) have computed the effects of support stiffness on the static and free vibration responses of a three-span simply supported plate. Also the critical buckling stresses are discussed. They have approached the problem by incorporating the finite strip method together with a spring system. (1) have investigated a particular case of the problem of free vibrations of a uniform beam with intermediate constraints, and the ends elastically restrained against rotation and translation. In (41), the fundamental modes of a free-vibrating beam, supported by two symmetrically placed elastic supports, have been analyzed in depth.

Multi-span beams and related problems have been considered by various authors. (15) have considered a cantilever beam with one or two additional lateral supports, and showed that there exists a minimum stiffness of the support above which the fundamental frequency of flexural vibrations of the beam no longer increases. (22) have considered free vibration analysis of a multispan beam with an arbitrary number of flexible constraints. They have assumed that each span of the continuous beam obeys Timoshenko beam theory, and they solve the system by the transfer matrix method. (35) have considered quasistatic bending problems of a multi-span beam on linear viscoelastic foundations of the Kelvin, Maxwell and standard linear solid types. They have solved the elastic free vibration problem, and then utilized the correspondence principle to determine the solution of the viscoelastic problem. They provide numerical results for a three-span beam. 
(32) have derived formulae for the computation of the response of periodically supported structures subject to a moving or stationary harmonic load, using a wavenumber-based approach. As a result, the load speed has been found to have a significant effect on the vibration of the conventional pinned-pinned ballasted track. (23) have considered free vibration analysis of a multi-span beam carrying multiple intermediate spring-mass systems. They have proposed an exact solution for the problem, and obtained the natural frequencies and associated mode shapes directly from the differential equation of motion of the continuous beam. (26) have considered the effect of a moving spring-mass system on the vibration of a cantilever beam.

Exact solutions to beam problems, in general, have been considered by multiple authors. (40) used a Green's function approach to derive exact solutions for beams of the Timoshenko type on an elastic foundation. A Green's function approach was also used in (12), concentrating on classical Euler-Bernoulli beams. The authors considered how the approach must be modified in order for a Green's function to exist, and under which circumstances it may occur that the problem has no solutions or an infinite number of solutions. (38) derived analytical solutions for circular beams made of functionally graded materials, concentrating on the in-plane static case.

A common element, in those of the above-mentioned studies that concentrate on axially moving materials, is that they focus on a single span. However, for example, a cylinder-based dryer section in a paper machine consists of a large number of spans. This number is typically about 70; see the book edited by (14). Furthermore, the rollers at the ends of the span are modelled as perfectly 
rigid, using the simply supported (pinned) boundary conditions for the transverse displacement of the paper web.

In the present study, we will investigate a simple fundamental problem where both of these assumptions are relaxed. We consider an infinite beam with identical supports placed at uniform intervals. This is an idealized model representing the middle part of a multi-span system, far from either of its ends. Furthermore, we model the supports as linearly elastic using Hooke's law. This, when compared to the case with rigid supports, allows us to study the effect of the elasticity of the supports on the vibration of the infinite beam.

For simplicity, we have chosen to neglect the axial motion of the material. Hence the quantitative results will be strictly applicable only to low-speed systems, where this motion can be neglected. Qualitative conclusions are expected to carry over also to high-speed systems.

We first set up the problem, and then derive closed-form analytical solutions for the frequencies of free vibrations and the corresponding eigenmodes. We conclude by asymptotic analyses of some periodic solutions.

\section{FORMULATION OF THE PROBLEM}

Let us consider an elastic continuous beam of unlimited length, elastically supported at the points $x_{n}=n \ell$, where $n=0, \pm 1, \pm 2 \ldots$, and the distance between two consecutive supports is denoted by $\ell$ (Figure 1). During small transverse vibrations, the position of the beam is sufficiently close 


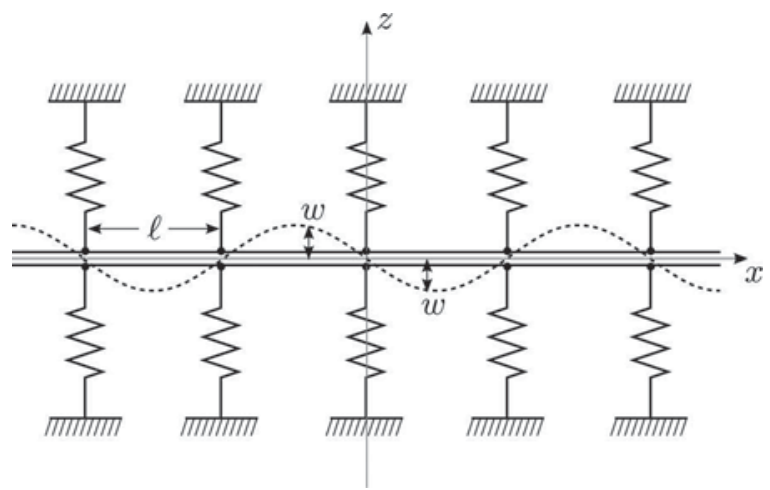

Figure 1: Periodic vibrations in the vicinity of equilibrium position for the beam of unlimited length with equidistant supports. Schematic representation.

to a position of stable equilibrium, and the transverse velocity will be small in absolute value. The reaction of the considered elastic supports is proportional to the deflection of the beam at that point, i.e. the beam reaction can be represented by Hooke's law, $k w$, in which $w$ is the deflection and $k$ is a constant usually called the rigidity modulus of the support (modulus of rigidity of an elastic string). In addition, it is supposed that the considered elastic point supports are characterized by the continuity conditions for displacements and its first derivative with respect to $x$. Such theoretical models for beams of unlimited length with an elastic system of supports are adequate to a wide class of engineering problems and have been discussed in the classical literature (see, for example, the books by (37) and (39)).

The governing equation for the deflection function $w(x)$, describing the free transverse vibrations in the interval $x_{n-1}<x<x_{n} n=0, \pm 1, \pm 2 \ldots$, is

$E I \frac{\mathrm{d}^{4} w}{\mathrm{~d} x^{4}}-\rho S \Omega^{2} w=0, \quad x_{n-1}<x<x_{n}$ 
with the following boundary conditions at the supporting points:

$$
\begin{aligned}
(w)_{x_{n}}^{+} & =(w)_{x_{n}}^{-}, \\
\left(\frac{\mathrm{d} w}{\mathrm{~d} x}\right)_{x_{n}}^{+} & =\left(\frac{\mathrm{d} w}{\mathrm{~d} x}\right)_{x_{n}}^{-}, \\
E I\left(\frac{\mathrm{d}^{2} w}{\mathrm{~d} x^{2}}\right)_{x_{n}}^{+} & =E I\left(\frac{\mathrm{d}^{2} w}{\mathrm{~d} x^{2}}\right)_{x_{n}}^{-}, \\
E I\left(\frac{\mathrm{d}^{3} w}{\mathrm{~d} x^{3}}\right)_{x_{n}}^{+}-E I\left(\frac{\mathrm{d}^{3} w}{\mathrm{~d} x^{3}}\right)_{x_{n}}^{-} & =-k(w)_{x_{n}}, \quad n=0, \pm 1, \pm 2, \ldots
\end{aligned}
$$

In (1)-(5), EI is the bending rigidity of the beam, $S$ the cross-sectional area of the beam, $\rho$ the density of the material, and $\Omega$ the frequency of harmonic vibrations. The superscripts \pm denote the limits of the corresponding values at $x_{n}+0$ and $x_{n}-0$. The condition (5) is Hooke's law for the elastic support located at $x_{n}$.

Note that the boundary conditions $(2)-(5)$ require only $C^{2}$ continuity at each support, with a finite jump in the third derivative. At this point in the analysis, no assumption is made of the possible periodicity of the solution.

For convenience, let us introduce the dimensionless quantities

$x=\ell \tilde{x}, \quad \omega^{2}=\rho \frac{S \ell^{4}}{E I} \Omega^{2}, \quad \gamma=\frac{k \ell^{3}}{E I}$. 
In the following, the tilde will be omitted. Using (6), the problem (1)-(5) can be rewritten in the form

$$
\begin{aligned}
\frac{\mathrm{d}^{4} w}{\mathrm{~d} x^{4}}-\omega^{2} w & =0, \quad x_{n-1}<x<x_{n} \\
(w)_{x_{n}}^{+} & =(w)_{x_{n}}^{-} \\
\left(\frac{\mathrm{d} w}{\mathrm{~d} x}\right)_{x_{n}}^{+} & =\left(\frac{\mathrm{d} w}{\mathrm{~d} x}\right)_{x_{n}}^{-}, \\
\left(\frac{\mathrm{d}^{2} w}{\mathrm{~d} x^{2}}\right)_{x_{n}}^{+} & =\left(\frac{\mathrm{d}^{2} w}{\mathrm{~d} x^{2}}\right)_{x_{n}}^{-}, \\
\left(\frac{\mathrm{d}^{3} w}{\mathrm{~d} x^{3}}\right)_{x_{n}}^{+}-\left(\frac{\mathrm{d}^{3} w}{\mathrm{~d} x^{3}}\right)_{x_{n}}^{-} & =-\gamma(w)_{x_{n}}, \quad n=0, \pm 1, \pm 2, \ldots
\end{aligned}
$$

Note that due to the dimensionless coordinates, each interval is of unit length.

To analyze the general properties of the spectral problem, we introduce the following notation:

$$
w_{n}=(w)_{x_{n}}, \quad \Theta_{n}=\left(\frac{\mathrm{d} w}{\mathrm{~d} x}\right)_{x_{n}}
$$

for the deflection and the derivative of the deflection at the points $x_{n}$, and $w^{n}$ for the deflection of the beam in the interval $x_{n-1} \leq x \leq x_{n}$, i.e. $w^{n}=w(x)$ if $x_{n-1} \leq x \leq x_{n}$. We will have

$$
\begin{array}{cl}
\left(w^{n}\right)_{x_{n-1}}=w_{n-1}, & \left(w^{n}\right)_{x_{n}}=w_{n} \\
\left(\frac{\mathrm{d} w^{n}}{\mathrm{~d} x}\right)_{x_{n-1}}=\Theta_{n-1}, & \left(\frac{\mathrm{d} w^{n}}{\mathrm{~d} x}\right)_{x_{n}}=\Theta_{n} .
\end{array}
$$

We can now represent the solution of the problem (7)-(11) in the interval $x_{n-1} \leq x \leq x_{n}$ in the form

$$
w^{n}=N_{11}^{n}(x) w_{n-1}+N_{12}^{n}(x) \Theta_{n-1}+N_{21}^{n}(x) w_{n}+N_{22}^{n}(x) \Theta_{n},
$$


where $N_{i j}^{n}(x), i, j=1,2$ are shape functions satisfying the differential equation (7). In correspondence with the definition of the node values $w_{n}$ and $\Theta_{n}$, the functions $N_{i j}^{n}(x)$ satisfy the following boundary conditions:

$$
\begin{array}{llll}
\left(N_{11}^{n}\right)_{x_{n-1}}=1, & \left(\frac{\mathrm{d} N_{11}^{n}}{\mathrm{~d} x}\right)_{x_{n-1}}=0, & \left(N_{11}^{n}\right)_{x_{n}}=0, & \left(\frac{\mathrm{d} N_{11}^{n}}{\mathrm{~d} x}\right)_{x_{n}}=0, \\
\left(N_{12}^{n}\right)_{x_{n-1}}=0, & \left(\frac{\mathrm{d} N_{12}^{n}}{\mathrm{~d} x}\right)_{x_{n-1}}=1, & \left(N_{12}^{n}\right)_{x_{n}}=0, & \left(\frac{\mathrm{d} N_{12}^{n}}{\mathrm{~d} x}\right)_{x_{n}}=0, \\
\left(N_{21}^{n}\right)_{x_{n-1}}=0, & \left(\frac{\mathrm{d} N_{21}^{n}}{\mathrm{~d} x}\right)_{x_{n-1}}=0, & \left(N_{21}^{n}\right)_{x_{n}}=1, & \left(\frac{\mathrm{d} N_{21}^{n}}{\mathrm{~d} x}\right)_{x_{n}}=0, \\
\left(N_{22}^{n}\right)_{x_{n-1}}=0, & \left(\frac{\mathrm{d} N_{22}^{n}}{\mathrm{~d} x}\right)_{x_{n-1}}=0, & \left(N_{22}^{n}\right)_{x_{n}}=0, & \left(\frac{\mathrm{d} N_{22}^{n}}{\mathrm{~d} x}\right)_{x_{n}}=1 .
\end{array}
$$

For brevity and simplicity we will use the vector notation

$$
\delta_{n}=\left\{\begin{array}{c}
w_{n} \\
\Theta_{n}
\end{array}\right\}, \quad N_{1}^{n}(x)=\left\{\begin{array}{c}
N_{11}^{n}(x) \\
N_{12}^{n}(x)
\end{array}\right\}, \quad N_{2}^{n}(x)=\left\{\begin{array}{l}
N_{21}^{n}(x) \\
N_{22}^{n}(x)
\end{array}\right\} .
$$

With the preceding notation, we may write

$$
\begin{aligned}
& w^{n}=\left(\delta_{n-1}, N_{1}^{n}(x)\right)+\left(\delta_{n}, N_{2}^{n}(x)\right), \\
& \left(\delta_{n-1},\left(\frac{\mathrm{d}^{2} N_{1}^{n}}{\mathrm{~d} x^{2}}\right)_{x_{n}}\right)+\left(\delta_{n},\left(\frac{\mathrm{d}^{2} N_{2}^{n}}{\mathrm{~d} x^{2}}\right)_{x_{n}}\right) \\
& \quad=\left(\delta_{n},\left(\frac{\mathrm{d}^{2} N_{1}^{n+1}}{\mathrm{~d} x^{2}}\right)_{x_{n}}\right)+\left(\delta_{n+1},\left(\frac{\mathrm{d}^{2} N_{2}^{n+1}}{\mathrm{~d} x^{2}}\right)_{x_{n}}\right), \\
& \left(\delta_{n-1},\left(\frac{\mathrm{d}^{3} N_{1}^{n}}{\mathrm{~d} x^{3}}\right)_{x_{n}}\right)+\left(\delta_{n},\left(\frac{\mathrm{d}^{3} N_{2}^{n}}{\mathrm{~d} x^{3}}\right)_{x_{n}}\right) \\
& \quad=\left(\delta_{n},\left(\frac{\mathrm{d}^{3} N_{1}^{n+1}}{\mathrm{~d} x^{3}}\right)_{x_{n}}\right)+\left(\delta_{n+1},\left(\frac{\mathrm{d}^{3} N_{2}^{n+1}}{\mathrm{~d} x^{3}}\right)_{x_{n}}\right),
\end{aligned}
$$


or in more compact matrix form,

$\mathbf{A} \delta_{n-1}+\mathbf{B} \delta_{n}+\mathbf{C} \delta_{n+1}=0, \quad n=0, \pm 1, \pm 2, \ldots$

where $\mathbf{A}, \mathbf{B}$ and $\mathbf{C}$ are real matrices $(2 \times 2)$, the elements of which are expressed with the help of the functions $N_{i j}^{n}(x)$ and their second and third derivatives at the nodes $x_{n}$.

In accordance with the methods of solution of the system of linear homogeneous finite-difference equations with constant coefficients, the fundamental solution of the considered system is found in the form

$\delta_{n}=d q^{n}$

where $d$ is a constant vector that does not depend on the index $n$, and $q$ is a complex-valued scalar. On the right-hand side, the superscript indicates exponentiation.

Taking into account that $n \rightarrow \pm \infty$, we conclude that a bounded solution of (20) in the form (21) will occur if and only if $|q|=1$. Consequently, we will take the following representation for the values $q$ :

$q=e^{i \alpha}, \quad 0 \leq \alpha \leq 2 \pi$

Here $\alpha$ is an arbitrary real number from the interval $[0,2 \pi)$. The vector $d$ is found as a result of substituting (21)-(22) into (20) and performing conventional calculations. 
Taking into account that the matrices $\mathbf{A}, \mathbf{B}$ and $\mathbf{C}$ are real, we note that the complex-conjugate expressions (marked by the superscript $*$ )

$\delta_{n}^{*}=d^{*}\left(q^{*}\right)^{n}=d^{*} e^{-i n \alpha}=d^{*} e^{i n(2 \pi-\alpha)}$

are also solutions apart from (21)-(22). Thus the solution $\delta_{n}^{*}$ is characterized by the parameter $\tilde{\alpha}=2 \pi-\alpha$.

Consider now the equivalent spectral problem formulated in the unit interval in the dimensionless variables. For this purpose, note the relation between the node values of the variables $w_{n}$ and $\Theta_{n}$ at the ends of the interval $x_{n-1} \leq x \leq x_{n}$ :

$\delta_{n}=\delta_{n-1} q$.

Using (24) and (17) we will have

$$
\begin{aligned}
w^{n+1} & =\left(\delta_{n}, N_{1}^{n+1}(x)\right)+\left(\delta_{n+1}, N_{2}^{n+1}(x)\right) \\
& =\left[\left(\delta_{n-1}, N_{1}^{n+1}(x)\right)+\left(\delta_{n}, N_{2}^{n+1}(x)\right)\right] q
\end{aligned}
$$

From representation (25) and the relations

$$
\left(\frac{\mathrm{d}^{s} N_{i j}^{n+1}}{\mathrm{~d} x^{s}}\right)_{x_{n}}=\left(\frac{\mathrm{d}^{s} N_{i j}^{n}}{\mathrm{~d} x^{s}}\right)_{x_{n-1}}, \quad s=0,1,2
$$

it follows that

$$
\left(\frac{\mathrm{d}^{s} w}{\mathrm{~d} x^{s}}\right)_{x_{n}}^{ \pm}=\left(\frac{\mathrm{d}^{s} w}{\mathrm{~d} x^{s}}\right)_{x_{n-1}}^{ \pm} q, \quad s=0,1,2
$$


The relations (26) require only that in each interval, the solution can be represented using the same set of basis functions, namely (14).

For the rest of this study, we will consider the unit interval $[0,1]$, and using the expression (22), we rewrite the relations $(27)$ in the form

$$
\left(\frac{\mathrm{d}^{s} w}{\mathrm{~d} x^{s}}\right)_{x=1}^{ \pm}=e^{i \alpha}\left(\frac{\mathrm{d}^{s} w}{\mathrm{~d} x^{s}}\right)_{x=0}^{ \pm}, \quad \alpha \in[0,2 \pi], \quad s=0,1,2 .
$$

Note that the relations (28), having the general nature, are valid for any periodic system.

The relations (28) can be used to represent the original multipoint periodic problem (7)-(11) in the form of a spectral problem, defined on the unit interval $[0,1]$, as

$$
\begin{aligned}
\frac{\mathrm{d}^{4} w}{\mathrm{~d} x^{4}}-\omega^{2} w & =0, \quad 0<x<1 \\
(w)_{x=1} & =(w)_{x=0} e^{i \alpha}, \quad\left(\frac{\mathrm{d} w}{\mathrm{~d} x}\right)_{x=1}=\left(\frac{\mathrm{d} w}{\mathrm{~d} x}\right)_{x=0} e^{i \alpha}, \quad\left(\frac{\mathrm{d}^{2} w}{\mathrm{~d} x^{2}}\right)_{x=1}=\left(\frac{\mathrm{d}^{2} w}{\mathrm{~d} x^{2}}\right)_{x=0} e^{i \alpha}, \\
\left(\frac{\mathrm{d}^{3} w}{\mathrm{~d} x^{3}}\right)_{x=1} & =\left[\left(\frac{\mathrm{d}^{3} w}{\mathrm{~d} x^{3}}\right)_{x=0}+\gamma(w)_{x=0}\right] e^{i \alpha}, \quad 0 \leq \alpha \leq 2 \pi .
\end{aligned}
$$

The spectral boundary value problem (29)-(31) is equivalent to the following variational problem:

$$
\omega^{2}(\alpha)=\min _{w_{\alpha} \in W_{\gamma}, w_{\alpha}^{*} \in W_{\gamma}^{*}}\left\{\frac{\int_{0}^{1} \frac{\mathrm{d}^{2} w_{\alpha}}{\mathrm{d} x^{2}} \frac{\mathrm{d}^{2} w_{\alpha}^{*}}{\mathrm{~d} x^{2}} \mathrm{~d} x+\gamma\left(w_{\alpha}\right)_{x=0}\left(w_{\alpha}^{*}\right)_{x=0}}{\int_{0}^{1} w_{\alpha} w_{\alpha}^{*} \mathrm{~d} x}\right\},
$$

where $W_{\gamma}$ and $W_{\gamma}^{*}$ are, respectively, the sets of functions satisfying the boundary conditions (30)(31) and the corresponding complex-conjugate conditions. 
The limiting case of absolutely rigid supports means that the value $\gamma=\infty$, and the deflection vanishes at the points $x=0$ and $x=1$. The corresponding boundary value problem takes the form

$$
\begin{aligned}
\frac{\mathrm{d}^{4} w}{\mathrm{~d} x^{4}}-\omega^{2} w & =0, \quad 0<x<1 \\
(w)_{x=0} & =0, \quad(w)_{x=1}=0, \quad\left(\frac{\mathrm{d} w}{\mathrm{~d} x}\right)_{x=1}=\left(\frac{\mathrm{d} w}{\mathrm{~d} x}\right)_{x=0} e^{i \alpha}, \\
\left(\frac{\mathrm{d}^{2} w}{\mathrm{~d} x^{2}}\right)_{x=1} & =\left(\frac{\mathrm{d}^{2} w}{\mathrm{~d} x^{2}}\right)_{x=0} e^{i \alpha}, \quad 0 \leq \alpha \leq 2 \pi
\end{aligned}
$$

In this case, the problem (33) is characterized by the following variational formulation:

$$
\omega^{2}(\alpha)=\min _{w_{\alpha} \in W_{r}, w_{\alpha}^{*} \in W_{r}^{*}}\left\{\frac{\int_{0}^{1} \frac{\mathrm{d}^{2} w_{\alpha}}{\mathrm{d} x^{2}} \frac{\mathrm{d}^{2} w_{\alpha}^{*}}{\mathrm{~d} x^{2}} \mathrm{~d} x}{\int_{0}^{1} w_{\alpha} w_{\alpha}^{*} \mathrm{~d} x}\right\} .
$$

Here $W_{r}$ and $W_{r}^{*}$ are, respectively, the admissible sets of functions satisfying the boundary conditions in (33) and the corresponding complex-conjugate boundary conditions.

The equations (29) and (33) play the role of necessary extremum conditions for the functionals (32) and (34), respectively. If we vary the functional (32), (34) and require that

$\delta \omega^{2}(\alpha)=0$

with corresponding boundary conditions, then we obtain the differential equations (29), (33) as Euler's equations of the functional.

From the relations (29)-(31), (33) it follows that the frequencies of harmonic vibrations $\omega^{2}=$ $\omega^{2}(\alpha)$ and the corresponding modes $w(x)=w_{\alpha}(x)$ depend continuously on the parameter $\alpha \in$ 
$[0,2 \pi]$. Consequently, the eigenvalues of the spectral problems (29)-(31), (33) fill continuous bands. For real eigenvalues $\omega^{2}=\omega^{2}(\alpha)$, apart from the eigenfunctions $w_{\alpha}(x)$, the complex conjugate functions $w_{\alpha}^{*}(x)$ also satisfy differential equations with complex conjugate boundary conditions. Taking into account that

$e^{-i \alpha}=e^{-i \alpha} e^{2 \pi i}=e^{i(2 \pi-\alpha)}$,

we obtain

$w_{\alpha}^{*}(x)=w_{2 \pi-\alpha}(x)$.

Note that for $\alpha=\pi$, we have

$w_{\pi}^{*}(x)=w_{\pi}(x)$,

and thus the solution $w_{\pi}(x)$ is real.

Using the variational representation for $\omega^{2}(2 \pi-\alpha)$ and the equality (37), we will have $\omega^{2}(\alpha)=$ $\omega^{2}(2 \pi-\alpha)$. As a result, we arrive at the conclusion that the two eigenfunctions $w_{\alpha}(x)$ and $w_{\alpha}^{*}(x)=w_{2 \pi-\alpha}(x)$ correspond to one and the same eigenvalue $\omega^{2}(\alpha)$. Consequently, for $\alpha \neq \pi$, the eigenfrequencies of harmonic vibrations are double and

$\omega^{2}(\alpha)=\omega^{2}(2 \pi-\alpha), \quad w_{\alpha}^{*}(x)=w_{2 \pi-\alpha}(x)$.

Using the properties (39), without loss of generality we can study the problem only for the interval $\alpha \in[0, \pi]$. 
Taking into account the properties of (29)-(31) and (33) with respect to the operation $x \rightarrow 1-x$, it is possible to perform more detailed analysis of the problem under consideration. With this purpose, we introduce the following notation:

$\tilde{w}_{\alpha}(x)=w_{\alpha}(1-x)$,

and note that the function $\tilde{w}_{\alpha}(x)$ apart from the function $w_{\alpha}(x)$ satisfies the differential equation for the one and the same value $\omega^{2}(\alpha)$. In this case the boundary conditions for the functions $\tilde{w}_{\alpha}(x)$ correspond to $\tilde{\alpha}=2 \pi-\alpha$ and in correspondence with (37) we have

$\tilde{w}_{\alpha}(x) \equiv w_{\alpha}^{*}(x)$

As a result, we obtain

$w_{\alpha}(1-x) \equiv w_{\alpha}^{*}(x), \quad 0 \leq x \leq 1, \quad 0 \leq \alpha<2 \pi$.

\section{CONSTRUCTION OF EXPLICIT SOLUTIONS}

Let us construct the solution of the spectral problem (7)-(11). To this purpose, we will represent the shape functions $N_{i j}^{n}(x)$ in the form

$$
\begin{aligned}
N_{i j}^{n}(x)= & A_{i j} \cosh \left(\sqrt{\omega}\left[x-x_{n-1}\right]\right)+B_{i j} \cos \left(\sqrt{\omega}\left[x-x_{n-1}\right]\right) \\
& +C_{i j} \sinh \left(\sqrt{\omega}\left[x-x_{n-1}\right]\right)+D_{i j} \sin \left(\sqrt{\omega}\left[x-x_{n-1}\right]\right) .
\end{aligned}
$$


The constants $A_{i j}, B_{i j}, C_{i j}$ and $D_{i j}$ are determined by inserting (43) into the boundary conditions (15), and solving the resulting equation system. The solution is

$$
\begin{aligned}
A_{11} & =(1-\cosh \sqrt{\omega} \cos \sqrt{\omega}-\sinh \sqrt{\omega} \sin \sqrt{\omega}) \Delta^{-1} \\
\Delta & =2(1-\cosh \sqrt{\omega} \cos \sqrt{\omega}) \\
A_{12} & =(\sinh \sqrt{\omega} \cos \sqrt{\omega}-\cosh \sqrt{\omega} \sin \sqrt{\omega})(\sqrt{\omega} \Delta)^{-1}, \\
A_{21} & =(\cosh \sqrt{\omega}-\cos \sqrt{\omega}) \Delta^{-1}, \quad A_{22}=-(\sinh \sqrt{\omega}-\sin \sqrt{\omega})(\sqrt{\omega} \Delta)^{-1}, \\
B_{11} & =(1-\cosh \sqrt{\omega} \cos \sqrt{\omega}+\sinh \sqrt{\omega} \sin \sqrt{\omega}) \Delta^{-1} \\
B_{12} & =-A_{12}, \quad B_{21}=-A_{21}, \quad B_{22}=-A_{22}, \\
C_{11} & =(\cosh \sqrt{\omega} \sin \sqrt{\omega}+\sinh \sqrt{\omega} \cos \sqrt{\omega}) \Delta^{-1}, \\
C_{12} & =(1-\cosh \sqrt{\omega} \cos \sqrt{\omega}+\sinh \sqrt{\omega} \sin \sqrt{\omega})(\sqrt{\omega} \Delta)^{-1}, \\
C_{21} & =-(\sinh \sqrt{\omega}+\sin \sqrt{\omega}) \Delta^{-1}, \quad C_{22}=(\cosh \sqrt{\omega}-\cos \sqrt{\omega})(\sqrt{\omega} \Delta)^{-1}, \\
D_{11} & =-C_{11}, \quad D_{21}=-C_{21}, \quad D_{22}=-C_{22}, \\
D_{12} & =(1-\cosh \sqrt{\omega} \cos \sqrt{\omega}-\sinh \sqrt{\omega} \sin \sqrt{\omega})(\sqrt{\omega} \Delta)^{-1} .
\end{aligned}
$$

Inserting (43)-(44) into (16)-(19), the matrices A, B and $\mathbf{C}$ in equation (20) can be represented as

$$
\mathbf{A}=\left(\begin{array}{cc}
\tilde{\alpha} \sqrt{\omega} & \beta \\
-\beta \sqrt{\omega} & -\tilde{\alpha}
\end{array}\right), \quad \mathbf{B}=\left(\begin{array}{cc}
0 & \gamma_{2} \\
\gamma_{1} \sqrt{\omega}+\gamma & 0
\end{array}\right), \quad \mathbf{C}=\left(\begin{array}{cc}
-\tilde{\alpha} \sqrt{\omega} & \beta \\
-\beta \sqrt{\omega} & -\tilde{\alpha}
\end{array}\right),
$$

where we have defined

$$
\begin{aligned}
\tilde{\alpha} & =2 \omega(\cosh \sqrt{\omega}-\cos \sqrt{\omega}) \Delta^{-1}, \quad \beta=2 \omega(\sinh \sqrt{\omega}-\sin \sqrt{\omega}) \Delta^{-1}, \\
\gamma_{1,2} & =4 \omega(\cosh \sqrt{\omega} \sin \sqrt{\omega} \pm \sinh \sqrt{\omega} \cos \sqrt{\omega}) \Delta^{-1}
\end{aligned}
$$

Substituting the expressions (45) into (20), we obtain a homogeneous system of linear equations for determining the components $d_{1}, d_{2}$ of the vector $d$ :

$$
\left[\begin{array}{cc}
-2 i \sqrt{\omega} \sin \alpha & 2 \beta \cos \alpha+\gamma_{2} \\
-2 \beta \sqrt{\omega} \cos \alpha+\gamma_{1} \sqrt{\omega}+\gamma & 2 i \tilde{\alpha} \sin \alpha
\end{array}\right]\left(\begin{array}{l}
d_{1} \\
d_{2}
\end{array}\right)=0 .
$$


To obtain a nontrivial solution of the system, we require that its determinant $\Delta(\omega ; \alpha, \gamma)$ vanishes:

$$
\begin{aligned}
\Delta(\omega ; \alpha, \gamma) \equiv & \cos ^{2} \alpha+\left[-(\cosh \sqrt{\omega}+\cos \sqrt{\omega})+\frac{\gamma}{4 \sqrt{\omega^{3}}}(\sinh \sqrt{\omega}-\sin \sqrt{\omega})\right] \cos \alpha \\
& +\cosh \sqrt{\omega} \cos \sqrt{\omega}+\frac{\gamma}{4 \sqrt{\omega^{3}}}(\cosh \sqrt{\omega} \sin \sqrt{\omega}-\sinh \sqrt{\omega} \cos \sqrt{\omega})=0
\end{aligned}
$$

Equation (47) determines the frequencies $\omega(\alpha, \gamma)$ of free harmonic vibrations of the continous beam as functions of rigidity $\gamma$ of supports and the parameter $\alpha$.

Let us analyze the solutions $\omega_{k}(\alpha, \gamma)$ of the equation

$\Delta(\omega ; \alpha, \gamma)=0$

Note that because of the identity $\cos \alpha \equiv \cos (2 \pi-\alpha)$ we have

$\omega(\alpha, \gamma) \equiv \omega(2 \pi-\alpha, \gamma), \quad 0 \leq \alpha \leq 2 \pi$

and consequently to analyze the solution of (48) it is enough to perform the analysis for the values $\alpha \in[0, \pi]$

\section{EXPLICIT SOLUTIONS WITH ZERO PHASE SHIFT}

In the next two sections, as an example, we will consider the behaviour of periodic solutions $\omega_{k}(\alpha, \gamma)$, first for zero phase shift per period, $\alpha=0$, and then with a phase inversion between adjacent periods, $\alpha=\pi$; i.e., we will consider solutions $\omega_{k}(0, \gamma)$ and $\omega_{k}(\pi, \gamma)$. Each of these two cases splits into two subcases by utilization of symmetry properties. 
In the case $\alpha=0$, the boundary value problem (29)-(31) is reduced to

$$
\begin{aligned}
\frac{\mathrm{d}^{4} w}{\mathrm{~d} x^{4}}-\omega^{2} w & =0, \quad 0<x<1 \\
(w)_{x=1} & =(w)_{x=0}, \quad\left(\frac{\mathrm{d} w}{\mathrm{~d} x}\right)_{x=1}=\left(\frac{\mathrm{d} w}{\mathrm{~d} x}\right)_{x=0}, \quad\left(\frac{\mathrm{d}^{2} w}{\mathrm{~d} x^{2}}\right)_{x=1}=\left(\frac{\mathrm{d}^{2} w}{\mathrm{~d} x^{2}}\right)_{x=0}, \\
\left(\frac{\mathrm{d}^{3} w}{\mathrm{~d} x^{3}}\right)_{x=1} & =\left(\frac{\mathrm{d}^{3} w}{\mathrm{~d} x^{3}}\right)_{x=0}+\gamma(w)_{x=0} .
\end{aligned}
$$

All relations in (50) are invariant with respect to the mirroring operation $x \rightarrow 1-x$ and consequently, all solutions of the spectral problem (50) can be classified using properties of symmetry:

$w^{s}(x) \equiv w^{s}(1-x)$

for symmetric solutions $w^{s}(x)$ and

$w^{a}(x) \equiv-w^{a}(1-x)$

for antisymmetric solutions $w^{a}(x)$.

For symmetric vibration modes, the boundary conditions of the problem (50) are rewritten as

$$
\begin{aligned}
& \left(\frac{\mathrm{d} w^{s}}{\mathrm{~d} x}\right)_{x=0}=0, \quad\left(\frac{\mathrm{d}^{3} w^{s}}{\mathrm{~d} x^{3}}\right)_{x=0}=-\frac{\gamma}{2}\left(w^{s}\right)_{x=0} \\
& \left(\frac{\mathrm{d} w^{s}}{\mathrm{~d} x}\right)_{x=1}=0, \quad\left(\frac{\mathrm{d}^{3} w^{s}}{\mathrm{~d} x^{3}}\right)_{x=1}=+\frac{\gamma}{2}\left(w^{s}\right)_{x=1} .
\end{aligned}
$$


Taking into account (53), we find the following representation for the symmetric modes:

$w^{s}(x)=C^{s}\left(\frac{\sin \left(\frac{\sqrt{\omega}}{2}\right)}{\sinh \left(\frac{\sqrt{\omega}}{2}\right)} \cosh \left(\sqrt{\omega}\left(x-\frac{1}{2}\right)\right)+\cos \left(\sqrt{\omega}\left(x-\frac{1}{2}\right)\right)\right)$,

and the transcendental equation for determining the frequencies corresponding to symmetric modes is

$\operatorname{coth} \frac{\sqrt{\omega}}{2}+\cot \frac{\sqrt{\omega}}{2}=\frac{4 \sqrt{\omega^{3}}}{\gamma}$

Let us denote by $\omega_{k}(\gamma)$ the $k$ th branch of the solution of equation (55). As the equation follows from a variational principle, it holds for all branches that

$\frac{\mathrm{d} \omega_{k}}{\mathrm{~d} \gamma} \geq 0$

Consequently $\omega_{k}$ are monotonically increasing functions and as a result we have

$\left(\omega_{k}\right)_{\gamma=0} \leq \omega_{k} \leq\left(\omega_{k}\right)_{\gamma=\infty}$

Figures 2 and 3 show the first two branches, $\omega_{0}^{s}$ and $\omega_{1}^{s}$, for the range $0 \leq \gamma \leq 10^{4}$. Note that

$\left.\omega_{0}^{s}\right|_{\gamma=\infty} \approx 22.3733,\left.\quad \omega_{1}^{s}\right|_{\gamma=\infty} \approx 120.9034$

and that the increase of $\omega_{0}^{s}$ and $\omega_{1}^{s}$ as functions of $\gamma$ is very slow.

Let us analyze equation (55) for asymptotically small $(\gamma \ll 1)$ and asymptotically large $(\gamma \gg 1)$ values of the parameter $\gamma$. Asymptotic representation of the solution of equation (55) for small $\gamma$ 


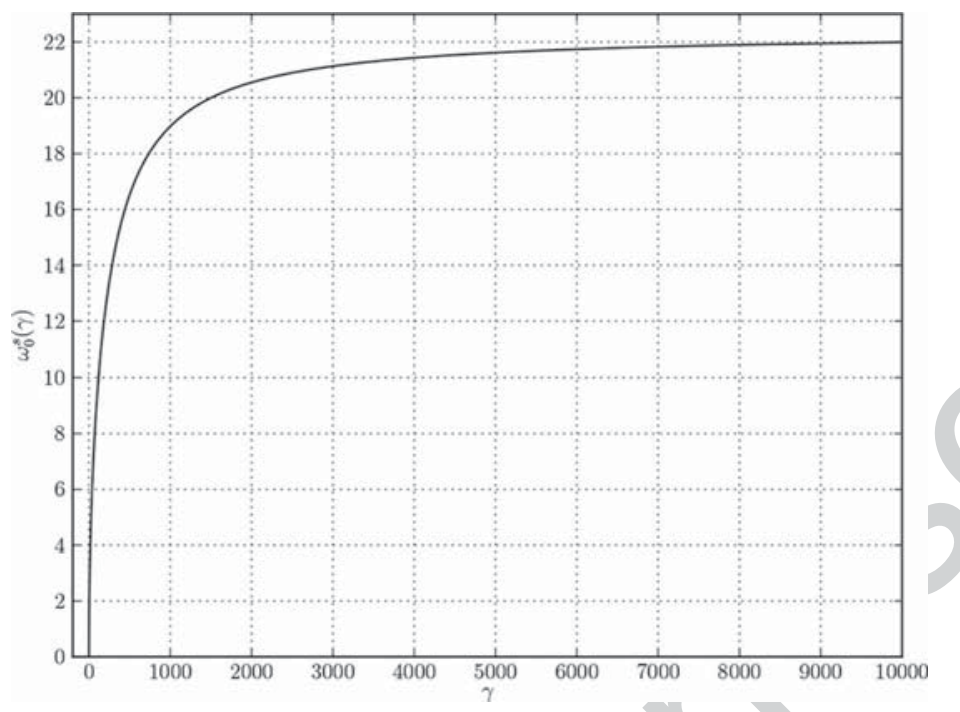

Figure 2: Frequency $\omega_{0}^{s}(\gamma)$ for $0<\gamma<10^{4}$.

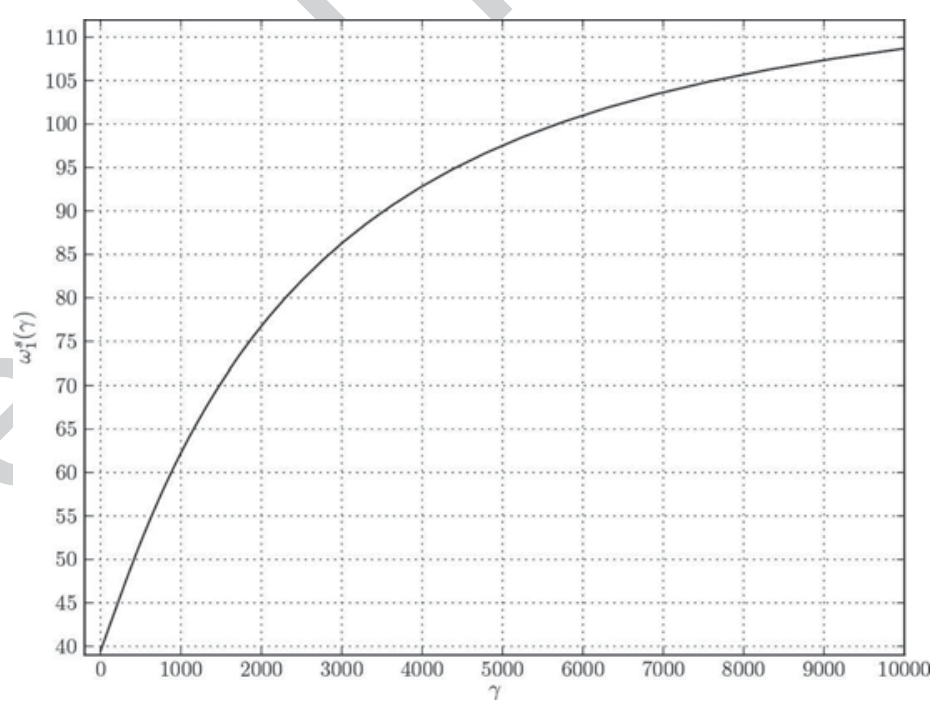

Figure 3: Frequency $\omega_{1}^{s}(\gamma)$ for $0<\gamma<10^{4}$. 
is given by the expression

$$
\omega_{k}^{s}(\gamma) \approx 4 k^{2} \pi^{2}+\frac{\gamma}{4 k^{2} \pi^{2}}, \quad k=1,2, \ldots
$$

The special case $\omega_{k}^{s}(\gamma) \ll 1$ is analyzed separately, taking also into account the smallness of $\gamma$. By series expansion around the point $\omega=0$, the left-hand side of (55) is approximately $4 / \sqrt{\omega}$, and hence we obtain

$\omega_{0}^{s}(\gamma) \approx \sqrt{\gamma}, \quad \gamma \ll 1$

Asymptotic solution (60) and rigorous numerical solutions of equation (55) practically coincide. The asymptotic representation (60) is very effective in the range $0 \leq \gamma \leq 10$. For example, for $\gamma=1$, we have that the rigorous numerical solution is $\omega_{0}^{s}(1)=0.999306$ and the asymptotic solution $\omega_{0}^{s}(1)=1$. For $\gamma=10$, the rigorous numerical solution gives $\omega_{0}^{s}(10)=3.14042$ and the asymptotic solution $\omega_{0}^{s}(10)=3.16228$; the values are within $1 \%$ of each other.

In the case $\gamma \rightarrow \infty$, equation (55) is reduced to

$\tan \left(\frac{\sqrt{\omega}}{2}\right)=-\tanh \left(\frac{\sqrt{\omega}}{2}\right)$

which gives the frequencies for the corresponding case with rigid supports. The first two solutions of (61), obtained numerically, were given in (58).

We observe that $\tanh (\sqrt{\omega} / 2) \rightarrow 1$ very quickly as $\omega$ increases; for $\omega \geq 2 \pi^{2}$, the difference is less than $3 \%$, and for $\omega \geq 4 \pi^{2}$, less than $0.3 \%$. This motivates an analytical approximation. Instead 
of (61), we solve $\tan (\sqrt{\omega} / 2)=-1$, obtaining

$\left.\omega_{0}^{s}\right|_{\gamma=\infty} \approx \frac{9}{4} \pi^{2} \approx 22.2066,\left.\quad \omega_{1}^{s}\right|_{\gamma=\infty} \approx \frac{49}{4} \pi^{2} \approx 120.9027$.

Using these approximations for the values $\omega_{k}^{s}(\gamma=\infty)$ and relations (59)-(60) for $\gamma \ll 1$, the inequalities (57) can be defined concretely, and written in the following form:

$4 k^{2} \pi^{2} \leq \omega_{k}^{s}(\gamma) \leq 4\left(k+\frac{3}{4}\right)^{2} \pi^{2}, \quad k=0,1,2, \ldots$,

For antisymmetric vibration modes $w^{a}(x)$, the boundary conditions in (50) take the following form:

$$
\begin{aligned}
& \left(w^{a}\right)_{x=0}=0, \quad\left(\frac{\mathrm{d}^{2} w^{a}}{\mathrm{~d} x^{2}}\right)_{x=0}=0, \\
& \left(w^{a}\right)_{x=1}=0, \quad\left(\frac{\mathrm{d}^{2} w^{a}}{\mathrm{~d} x^{2}}\right)_{x=1}=0 .
\end{aligned}
$$

In this case, the solutions of the spectral problem with boundary conditions (63) coincide with the antisymmetric solutions of the harmonic vibration problem for the beam with hinged supports. We have

$\omega_{k}^{a}=4 k^{2} \pi^{2}, \quad k=1,2, \ldots$

$w_{k}^{a}(x)=C_{k}^{a} \sin \left(2 k \pi\left[x-\frac{1}{2}\right]\right)$.

Thus, this set of solutions does not depend on the rigidity of the supports. 


\section{EXPLICIT SOLUTIONS WITH INVERTING PHASE SHIFT}

Let us move onto the case $\alpha=\pi$. The boundary value problem (29)-(31) is reduced to

$$
\begin{aligned}
\frac{\mathrm{d}^{4} w}{\mathrm{~d} x^{4}}-\omega^{2} w & =0, \quad 0<x<1 \\
(w)_{x=1} & =-(w)_{x=0}, \quad\left(\frac{\mathrm{d} w}{\mathrm{~d} x}\right)_{x=1}=-\left(\frac{\mathrm{d} w}{\mathrm{~d} x}\right)_{x=0}, \quad\left(\frac{\mathrm{d}^{2} w}{\mathrm{~d} x^{2}}\right)_{x=1}=-\left(\frac{\mathrm{d}^{2} w}{\mathrm{~d} x^{2}}\right)_{x=0} \\
\left(\frac{\mathrm{d}^{3} w}{\mathrm{~d} x^{3}}\right)_{x=1} & =-\left(\frac{\mathrm{d}^{3} w}{\mathrm{~d} x^{3}}\right)_{x=0}-\gamma(w)_{x=0}
\end{aligned}
$$

The problem (65) is invariant with respect to the transformation $x \rightarrow 1-x$. Consequently, all solutions of the problem (65) are classified as either symmetric vibration modes $w^{s}(x)$, for which $w^{s}(x)=w^{s}(1-x)$, or antisymmetric vibration modes $w^{a}(x)$, for which $w^{a}(x)=-w^{a}(1-x)$.

It follows from (65) that the boundary conditions for symmetric modes $w^{s}(x)$ have the form

$$
\begin{aligned}
& \left(w^{s}\right)_{x=0}=0, \quad\left(\frac{\mathrm{d}^{2} w^{s}}{\mathrm{~d} x^{2}}\right)_{x=0}=0 \\
& \left(w^{s}\right)_{x=1}=0, \quad\left(\frac{\mathrm{d}^{2} w^{s}}{\mathrm{~d} x^{2}}\right)_{x=1}=0
\end{aligned}
$$

These modes correspond to the symmetric solutions of the harmonic vibration problem for the beam with hinged supports.

The boundary conditions (66), at first glance, may look the same as (63). The difference is implicit in the nature of symmetric and antisymmetric solutions. Symmetric solutions will automatically satisfy the boundary condition for $\mathrm{d} w / \mathrm{d} x$ in (65). The condition $\left(w^{s}\right)_{x=0}=0$ eliminates the $\gamma$ term 
from the boundary condition for $\mathrm{d}^{3} w / \mathrm{d} x^{3}$, and the remaining part of this condition is automatically satisfied because the solution is symmetric.

Similarly, in the previous case (63), the antisymmetric nature of the solution will automatically satisfy the condition for $\mathrm{d} w / \mathrm{d} x$ set in (50), while the condition $\left(w^{a}\right)_{x=0}=0$ again eliminates the $\gamma$ term from the boundary condition for $\mathrm{d}^{3} w / \mathrm{d} x^{3}$. The remaining part of that condition is then automatically satisfied because the solution is antisymmetric.

Returning to the symmetric solutions for (65), in this case we have

$\omega_{k}^{s}=(2 k+1)^{2} \pi^{2}, \quad k=0,1,2, \ldots$

$w_{k}^{s}(x)=C_{k}^{s} \cos \left((2 k+1) \pi\left[x-\frac{1}{2}\right]\right)$.

This set of solutions does not depend on the rigidity of the supports.

For antisymmetric vibration modes $w^{a}(x)$, we will have the following boundary conditions:

$$
\begin{aligned}
& \left(\frac{\mathrm{d} w^{a}}{\mathrm{~d} x}\right)_{x=0}=0, \quad\left(\frac{\mathrm{d}^{3} w^{a}}{\mathrm{~d} x^{3}}\right)_{x=0}=-\frac{\gamma}{2}\left(w^{a}\right)_{x=0}, \\
& \left(\frac{\mathrm{d} w^{a}}{\mathrm{~d} x}\right)_{x=1}=0, \quad\left(\frac{\mathrm{d}^{3} w^{a}}{\mathrm{~d} x^{3}}\right)_{x=1}=+\frac{\gamma}{2}\left(w^{a}\right)_{x=1} .
\end{aligned}
$$

The above remark on the boundary conditions applies also to the difference between the cases (68) and (53). 


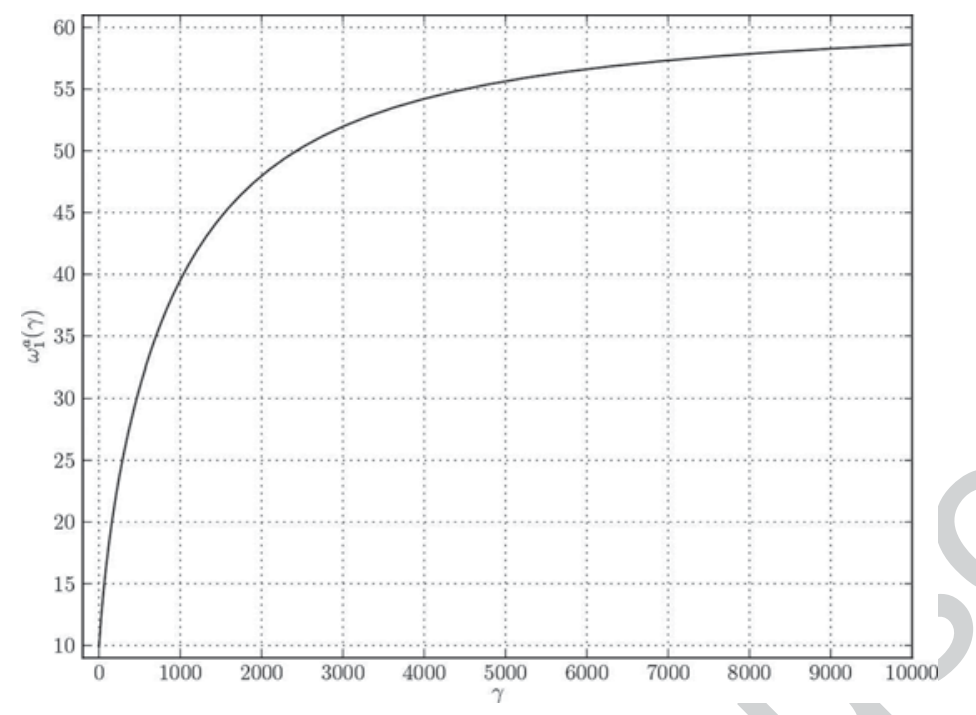

Figure 4: Frequency $\omega_{1}^{a}(\gamma)$ for $0<\gamma<10^{4}$.

In this case we find the following shape of vibrations:

$w^{a}(x)=C^{a}\left(-\frac{\cos \left(\frac{\sqrt{\omega}}{2}\right)}{\cosh \left(\frac{\sqrt{\omega}}{2}\right)} \sinh \left(\sqrt{\omega}\left[x-\frac{1}{2}\right]\right)+\sin \left(\sqrt{\omega}\left[x-\frac{1}{2}\right]\right)\right)$,

and the following transcendental equation for their frequencies:

$\tanh \left(\frac{\sqrt{\omega}}{2}\right)-\tan \left(\frac{\sqrt{\omega}}{2}\right)=\frac{4 \sqrt{\omega^{3}}}{\gamma}$

See Figures 4 and 5 for the first two branches, $\omega_{1}^{a}$ and $\omega_{2}^{a}$, for the range $0 \leq \gamma \leq 10^{4}$.

If $\gamma \ll 1$, then the solutions of equation (70) have the following asymptotic representation:

$\omega_{k}^{a}(\gamma)=(2 k-1)^{2} \pi^{2}+\frac{\gamma}{(2 k-1)^{2} \pi^{2}}, \quad k=1,2, \ldots$ 


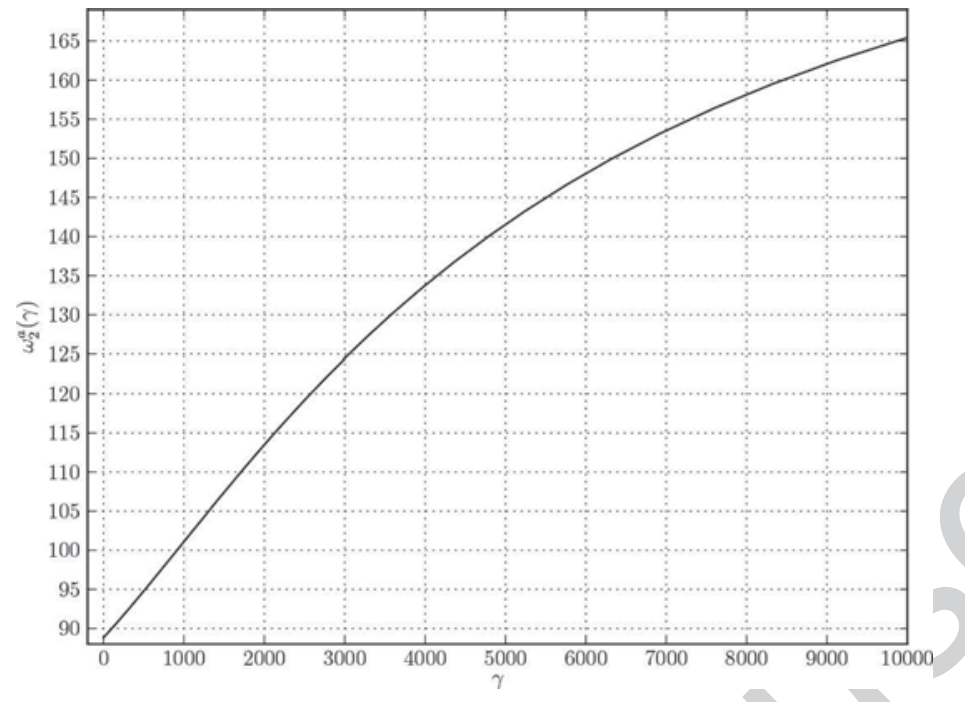

Figure 5: Frequency $\omega_{2}^{a}(\gamma)$ for $0<\gamma<10^{4}$.

Note that, in contrast to the case with symmetric vibrations, in (71) there are no frequencies which tend to zero. In the case $\gamma \rightarrow \infty$, the equation (70) is reduced to

$\tan \left(\frac{\sqrt{\omega}}{2}\right)=\tanh \left(\frac{\sqrt{\omega}}{2}\right)$

and the corresponding modes have the form

$w^{a}(x)=C^{a}\left(-\frac{\sin \left(\frac{\sqrt{\omega}}{2}\right)}{\sinh \left(\frac{\sqrt{\omega}}{2}\right)} \sinh \left(\sqrt{\omega}\left[x-\frac{1}{2}\right]\right)+\sin \left(\sqrt{\omega}\left[x-\frac{1}{2}\right]\right)\right)$.

The relations (72)-(73) describe the antisymmetric vibrations of the continuous beam with clamped ends. The first two solutions of (72), obtained numerically, are

$\left.\omega_{1}^{a}\right|_{\gamma=\infty} \approx 61.6728,\left.\quad \omega_{2}^{a}\right|_{\gamma=\infty} \approx 199.8594$ 
Taking into account (71) and the following approximate values for $\omega_{k}^{a}(\gamma=\infty)$ obtained by solving the approximate equation $\tan (\sqrt{\omega} / 2)=1$ :

$\left.\omega_{1}^{a}\right|_{\gamma=\infty} \approx \frac{25 \pi^{2}}{4} \approx 61.6850,\left.\quad \omega_{2}^{a}\right|_{\gamma=\infty} \approx \frac{81 \pi^{2}}{4} \approx 199.8595$,

we find the following two-sided estimate for the frequencies $\omega_{k}^{a}(\gamma)$ :

$(2 k-1)^{2} \pi^{2} \leq \omega_{k}^{a}(\gamma) \leq\left.\omega_{k}^{a}\right|_{\gamma=\infty} \approx\left(2 k+\frac{1}{2}\right)^{2} \pi^{2}, \quad k=1,2, \ldots$

\section{CONCLUSION}

In this paper, the problem of harmonic vibrations of a continuous infinite elastic beam having constant rigidity and inertial characteristics was investigated. The beam was fastened by a set of equally spaced elastic supports.

Closed-form analytical solutions were obtained for the frequencies of free vibrations and the corresponding eigenmodes. The transcendental equations determining the frequencies, appearing in two of the four cases studied in detail, were solved numerically.

It was observed that the frequencies increase monotonically as the rigidity of the supports is increased. For each mode, the frequency is bounded from above by a limiting value, which is realized for the case with absolutely rigid supports. For these upper limits, both numerical solutions and analytical approximations were given. 
The frequencies are also bounded from below by the limit solution as the rigidity of the supports approaches zero. Asymptotic representations were given for these lower limits.

The motivation and context of the study is in the process industry, especially paper making. The aim was to relax two common assumptions: those of a single span, and of absolutely rigid supports. The infinite beam considered in this study is a simplified model representing the middle part of a multi-span system, such as a cylinder-based dryer section, far from either of its ends. The beam model is appropriate, as it can also be used to model cylindrical deformation of a thin sheet.

Because in this study, the axial motion is neglected, the results are strictly quantitatively valid only for low-speed axially moving systems. However, the qualitative results are expected to carry over also to high-speed systems; it is expected that similar lower and upper bounds still apply. For quantitative results for high-speed systems, future studies are required.

Another limitation is that the study concentrated on the case of identical supports, with each support having the same boundary conditions. Although this is the most relevant case in a process industry context, mathematical generalization into supports with inequal stiffnesses is a possible topic for further studies.

In conclusion, we have seen that the elasticity in the supports, which allows them to vibrate, reduces the natural frequencies of the beam when compared to the case of absolutely rigid supports. Thus 
one may expect that in a real system, the natural frequencies will be lower than those predicted by a model with absolutely rigid supports.

\section{ACKNOWLEDGEMENT}

This research was supported by the Academy of Finland (Grant no. 269351); by the Program for RFBR (Grant 11-08-00030-a); by the RAS program 12, Program of Supports of Leading Scientific Schools (Grant 2611.2012.1); by the Finnish Cultural Foundation; and by the Jenny and Antti Wihuri Foundation.

\section{REFERENCES}

1. Albarracan, C. M., Zannier, L., Grossi, R. O. (2004). Some observations in the dynamics of beams with intermediate supports. Journal of Sound and Vibration 271(1-2):475-480.

2. Archibald, F. R., Emslie, A. G. (1958). The vibration of a string having a uniform motion along its length. ASME Journal of Applied Mechanics 25:347-348.

3. Banichuk, N., Jeronen, J., Kurki, M., Neittaanmäki, P., Saksa, T., Tuovinen, T. (2011). On the limit velocity and buckling phenomena of axially moving orthotropic membranes and plates. International Journal of Solids and Structures, 48(13):2015-2025.

4. Banichuk, N., Jeronen, J., Neittaanmäki, P., Saksa, T., Tuovinen, T. (2013a). Theoretical study on travelling web dynamics and instability under non-homogeneous tension. International Journal of Mechanical Sciences, 66C:132-140.

5. Banichuk, N., Jeronen, J., Neittaanmäki, P., Saksa, T., Tuovinen, T. (2014). Mechanics of Moving Materials. Vol. 207. Solid mechanics and its applications. Springer. 
6. Banichuk, N., Jeronen, J., Neittaanmäki, P., Tuovinen, T. (2010a). On the instability of an axially moving elastic plate. International Journal of Solids and Structures 47(1):91-99.

7. Banichuk, N., Jeronen, J., Neittaanmäki, P., Tuovinen, T. (2010b). Static instability analysis for travelling membranes and plates interacting with axially moving ideal fluid. Journal of Fluids and Structures 26(2):274-291.

8. Banichuk, N., Kurki, M., Neittaanmäki, P., Saksa, T., Tirronen, M., Tuovinen, T. (2013b). Optimization and analysis of processes with moving materials subjected to fatigue fracture and instability. Mechanics Based Design of Structures and Machines: An International Journal 41(2):146-167.

9. Bhat, R. B., Xistris, G. D., Sankar, T. S. (1982). Dynamic behavior of a moving belt supported on elastic foundation. Journal of Mechanical Design 104(1):143-147.

10. Chen, L.-Q. (2005). Analysis and control of transverse vibrations of axially moving strings. ASME Applied Mechanics Reviews 58:91-116.

11. Ghayesh, M. H., Amabili, M. (2013). Parametric stability and bifurcations of axially moving viscoelastic beams with time-dependent axial speed. Mechanics Based Design of Structures and Machines: An International Journal 41(3):359-381.

12. Hozhabrossadati, S. M., Sani, A. A. (2015). Deformation of Euler-Bernoulli beams by means of modified Green's function: Application of Fredholm alternative theorem. Mechanics Based Design of Structures and Machines: An International Journal, 43(3):277-293.

13. Huang, M.-H., Thambiratnam, D. P. (2001). Analysis of plate resting on elastic supports and elastic foundation by finite strip method. Computers and Structures 79(29-30):2547-2557. 
14. Karlsson, M., Ed. (2000). Papermaking Science and Technology. Vol. 9: Papermaking Part 2: Drying. Helsinki, Finland: Fapet Oy.

15. Kesson, B., Olhoff, N. (1988). Minimum stiffness of optimally located supports for maximum value of beam eigenfrequencies. Journal of Sound and Vibration 120(3):457-463.

16. Kong, L., Parker, R. G. (2004). Approximate eigensolutions of axially moving beams with small flexural stiffness. Journal of Sound and Vibration 276:459-469.

17. Li, W. L. (2000). Free vibrations of beams with general boundary conditions. Journal of Sound and Vibration 237(4):709-725.

18. Li, W. L. (2001). Dynamic analysis of beams with arbitrary elastic supports at both ends. Journal of Sound and Vibration 246(4):751-756.

19. Li, W. L. (2002). Reply to: Discussion on free vibrations of beams with general boundary conditions. Journal of Sound and Vibration 257(3):593-595.

20. Li, W. L. (2004). Vibration analysis of rectangular plates with general elastic boundary supports. Journal of Sound and Vibration 273(3):619-635.

21. Lin, C. C. (1997). Stability and vibration characteristics of axially moving plates. International Journal of Solids and Structures 34(24):3179-3190.

22. Lin, H.-P., Chang, S. C. (2005). Free vibration analysis of multi-span beams with intermediate flexible constraints. Journal of Sound and Vibration 281(1-2):155-169.

23. Lin, H.-Y., Tsai, Y.-C. (2007). Free vibration analysis of a uniform multi-span beam carrying multiple spring-mass systems. Journal of Sound and Vibration 302(3):442-456.

24. Marynowski, K., Kapitaniak, T. (2014). Dynamics of axially moving continua. International Journal of Mechanical Sciences 81:26-41. 
25. Miranker, W. L. (1960). The wave equation in a medium in motion. IBM Journal of Research and Development 4:36-42.

26. Mohebpour, S. R., Daneshmand, F., Mehregan, H. A. (2013). Numerical analysis of inclined flexible beam carrying one degree of freedom moving mass including centrifugal and Coriolis accelerations and rotary inertia effects. Mechanics Based Design of Structures and Machines: An International Journal 41(2):123-145.

27. Mote, C. D. (1972). Dynamic stability of axially moving materials. Shock and Vibration Digest $4(4): 2-11$.

28. Parker, R. G. (1998). On the eigenvalues and critical speed stability of gyroscopic continua. ASME Journal of Applied Mechanics 65:134-140.

29. Parker, R. G. (1999). Supercritical speed stability of the trivial equilibrium of an axiallymoving string on an elastic foundation. Journal of Sound and Vibration 221(2):205-219.

30. Perkins, N. C. (1990). Linear dynamics of a translating string on an elastic foundation. Journal of Vibration and Acoustics 112(1):2-7.

31. Sack, R. A. (1954). Transverse oscillations in traveling strings. British Journal of Applied Physics 5:224-226.

32. Sheng, X., Jones, C. J. C., Thompson, D. J. (2005). Responses of infinite periodic structures to moving or stationary harmonic loads. Journal of Sound and Vibration 282(1-2):125-149.

33. Simpson, A. (1973). Transverse modes and frequencies of beams translating between fixed end supports. Journal of Mechanical Engineering Science 15:159-164.

34. Skutch, R. (1897). Uber die Bewegung eines gespannten Fadens, weicher gezwungen ist durch zwei feste Punkte, mit einer constanten Geschwindigkeit zu gehen, und zwischen denselben 
in Transversal-schwingungen von gerlinger Amplitude versetzt wird. Annalen der Physik und Chemie 61:190-195.

35. Sonoda, K., Kobayashi, H. (1981). Multispan beams on linear viscoelastic foundations. Journal of Structural Mechanics 9(4):451-464.

36. Swope, R. D., Ames, W. F. (1963). Vibrations of a moving threadline. Journal of the Franklin Institute 275:36-55.

37. Timoshenko, S. P., Woinowsky-Krieger, S. (1959). Theory of Plates and Shells. 2nd ed. New York, Tokyo: McGraw-Hill.

38. Tufekci, E., Eroglu, U., Aya, S. A. (2016). Exact solution for in-plane static problems of circular beams made of functionally graded materials. Mechanics Based Design of Structures and Machines: An International Journal 44(4):476-494.

39. von Kármán, T., Biot, M. A. (1940). Mathematical Methods in Engineering. McGraw-Hill.

40. Wang, C. M., Lam, K. Y., He, X. Q. (1998). Exact solutions for Timoshenko beams on elastic foundations using Green's functions. Mechanics of Structures and Machines 26(1):101-113.

41. Wang, C. Y. (2003). Fundamental frequency of a beam on two elastic supports. Journal of Sound and Vibration 259(3):711-714.

42. Wickert, J. A. (1992). Non-linear vibration of a traveling tensioned beam. International Journal of Non-Linear Mechanics 27(3):503-517.

43. Wickert, J. A. (1994). Response solutions for the vibration of a traveling string on an elastic foundation. Journal of Vibration and Acoustics 116(1):137-139.

44. Wickert, J. A., Mote, C. D. (1990). Classical vibration analysis of axially moving continua. ASME Journal of Applied Mechanics 57:738-744. 